\title{
Interkulturelle Theologie als herausfordernde Ressource im Religionsunterricht
}

\author{
Britta Konz
}

\begin{abstract}
In the current situation of globalization and migration, migration societies are increasingly becoming culturally and religiously more diverse. As a result, interreligious learning processes in religious education have to be initiated. At the same time the reality of Christian internal differentiation and Christianity's own cultural diversity should also be taken into consideration. Today's challenge is to build a Christian identity that does not fall back into "traditional patterns of confessionalization", but rather unfolds religious diversity and intra-religious plurality. However, intercultural theology and postcolonial theory are still not sufficiently taken into account in religious education. Questions relating to migration society are predominantly negotiated in the field of interreligious learning. Therefore, this article examines postcolonial theory and intercultural theology, showing possible ways in which intra-religious learning can be shaped and the search for cultural-religious interspaces can be encouraged. According to the essay's main theory intercultural theology confronts us with contrasting religious interpretations, conceptions and practices and thus becomes a challenge, but also a resource for the shaping of religious identity in the context of migration and globalization. Intercultural theology challenges us to develop a denominational selfunderstanding which is not based on 'religious othering' or on intra-religious othering.
\end{abstract}

\section{Ausgangspunkt: Inklusion fördern im Kontext von Globalisierung und Migration}

Moderne Gesellschaften sind durch ethnische, kulturelle und religiöse Pluralität gekennzeichnet. Zum einen bestimmt Migration, wenn auch regional unterschiedlich ausgeformt, die aktuellen geschichtlichen Entwicklungen. ${ }^{1}$

1 Vgl. EKD, Zusammenleben gestalten. Ein Beitrag des Rates der EKD zu Fragen der Integration und des Zusammenlebens mit Menschen anderer Herkunft, Sprache oder Religion, EKD-T 76, 2002, http://www.ekd.de/EKD-Texte/ekd_texte_76_1.html. 
Deutschland ist dadurch, wie viele andere europäische Länder, in der zweiten Hälfte des 20. Jahrhunderts faktisch zu einer Migrationsgesellschaft mit kultureller und religiöser Vielfalt geworden. ${ }^{2}$ Zum anderen werden auch infolge der „Globalisierung“ die „Interdependenzen zwischen Nahem und Fernem und zwischen Lokalem und Globalem verdichtet ${ }^{\text {“3 }}$. Durch die wachsenden weltweiten Verflechtungen auf verschiedenen Ebenen ereignen sich Gesellschaft und damit auch schulische Bildung heute „zwangsläufig in einem globalen Referenzrahmen“4. Das „Lernen im Horizont der Weltgesellschaft“ muss folglich grundlegend neu verortet werden, ${ }^{5}$ damit das Zusammenleben von Menschen unterschiedlicher kultureller, religiöser und weltanschaulicher Orientierungen gut gestaltet werden kann, auch in Bezug auf ein Nebeneinander religiöser und nicht-religiöser Überzeugungen. ${ }^{6}$ Es müssen Bildungsprozesse angebahnt werden, mittels derer Heranwachsende lernen, „die intellektuellen und ethischen Potenziale der Menschen zu entfalten, derer die Weltgesellschaft für eine kooperative Bewältigung der globalen Risiken bedarf"7. Immer noch zu sehr überwiegt, so Seitz, im gesellschaftlichen Diskurs „ein dem Kosmopolitismus der Aufklärung zugrundeliegender exklusiver Universalismus", der sich durch die Negation des „Anderen der Vernunft“ konstituiert, durch die Verdrängung und Ausgrenzung von allem, was fremd und irrational erscheint, wie z.B. die „Fremdheit" anderer Kulturkreise. ${ }^{8}$ Der „Umgang mit Komplexität" ist deshalb eine Schlüsselkompetenz für das 21. Jahrhundert. ${ }^{9}$

2 Martin Affolderbach, Migration, Religion und Bildung - national und international, in: Peter Schreiner et AL. (Hg.), Handbuch Interreligiöses Lernen, Gütersloh 2005, 70-84, 70 u. 72.

3 Klaus Seitz, Bildung im Horizont der Weltgesellschaft. Weltbürgerliche Erziehung im Zeitalter der Globalisierung, in:ТномAs SCHREIJÄCK (Hg.), Christwerden im Kulturwandel. Analysen, Themen und Optionen für Religionspädagogik und Praktische Theologie. Ein Handbuch, Freiburg im Breisgau 2001, 47-77, 49 .

4 A.a.O., 50.

5 Ebd.

6 Positionspapier Religionspädagogik (http://akrk.eu/positionspapier-damit-der-religionsunterricht-in-deutschland-zukunftsfaehig-bleibt/) (10.1.2017) u. vgl. EKD-Denkschrift, Religiöse Orientierung gewinnen. Evangelischer Religionsunterricht als Beitrag zu einer pluralitätsfähigen Schule, November 2014 https://www.ekd.de/EKD-Texte/herausforderungen_ religioes_weltanschaulichen_vielfalt.html.

7 Seitz, Bildung, 76 .

8 A.a.O., 71 .

9 Ebd. 
Die Frage danach, wie man angemessen mit Heterogenität verfährt, ist seit über zwanzig Jahren Gegenstand theoretischer Diskurse, pädagogischer Weisungen und von Aus-und Fortbildungen von Lehrerinnenund Lehrern. ${ }^{10}$ Infolge der2oog in Kraft getretenen UN-Behindertenrechtskonvention ist Deutschland in der Pflicht, schulische Inklusion umzusetzen und hierfür tragende Konzepte zu entwickeln. Hierbei wird die Forderung nach Inklusion oft fälschlicherweise allein auf die Inklusion von Schülerinnen und Schülern mit Behinderung reduziert. Inklusion intendiert tatsächlich aber insgesamt eine gerechte Gesellschaft, in der niemand aufgrund von „Rasse“, Klasse, sexueller Orientierung, Religion, Geschlecht oder aufgrund von so genannter Behinderung ausgeschlossen wird. Dementsprechend wird in einer inklusiven Pädagogik der Vielfalt auch die „Heterogenitätsdimension der, sozialen Lage' oder des, sozialen Milieus“" in den Bick genommen. ${ }^{11}$ Auch der globale Horizont der Inklusionsforderungen darf in der Diskussion nicht vernachlässigt werden, vor allem, weil er infolge von Migration und Flucht immer mehr zur schulischen Realität auch in Deutschland wird. Laut Unesco sind vor allem Flüchtlinge, Nomadenkinder, Kinder mit Behinderung, missbrauchte Kinder, arbeitende Kinder, religiöse und ethnische Minderheiten, Migranten, Straßenkinder, Mädchen und Frauen, linguistische Minderheiten, Kindersoldaten, Landbevölkerung und HIV/AidsWaisen vom strukturellen Ausschluss aus dem Bildungssystem bedroht. ${ }^{12}$

Im Hinblick auf die vielfältigen kulturellen und religiösen Pluralisierungsprozesse sind Schule und Religionsunterricht dazu herausgefordert, interkulturelleund interreligiöseBildungs-undVerständigungsprozesse einzuleiten, die auf ein "Miteinander der Verschiedenen“ zielen. ${ }^{13}$ Sowohl von Lehrerinnen und Lehrern als auch von Schülerinnen und Schülern ist in zunehmendem Maße Diversitätskompetenz und Pluralitätswürdigung

10 MARIA Kron, Ausgangspunkt: Heterogenität. Weg und Ziel: Inklusion? Reflexionen zur Situation im Elementarbereich, Inklusion online-net 3/2010. In ihrem Aufsatz spricht Kron von einer fast 20-jährigen Diskussion. http://www.inklusion-online.net/index.php/ inklusion-online/article/view/120/120 (20.1.2017).

11 Dörthe Vieregge, „Wenn der Typ korrekt ist, dann gucke ich nicht drauf, welche Religion er hat." Deutungen sozial benachteiligter Jugendlicher zum Umgang mit religiöser Differenz in der Lebenswelt, in: Katharina Kammeyer et AL. (Hg.), Inklusion und Kindertheologie, Inklusion - Religion - Bildung 1, Münster 2014, 82-93, 92.

12 Vgl. Saskia Flake/Ina Schröder, Inklusive Pädagogik - Eine Herausforderung für die Religionspädagogik?!, in: Katharina Kammeyer et Al. (Hg.), Inklusion und Kindertheologie, Inklusion - Religion - Bildung 1, Münster 2014, 30-64, 42.

13 Anita Müller-Friese, Miteinander der Verschiedenen. Theologische Überlegungen zu einem integrativen Bildungsverständnis, FPDR 13, Weinheim 1996. 
gefordert. Soll Inklusion gelingen, darf jedoch keine unreflektierte „Celebration of diversity“ vollzogen werden. ${ }^{14}$ Stattdessen muss sich die Pädagogik um „einen aufgeklärten Umgang mit Kategorien“ bemühen und nicht nur die materiellen Barrieren, sondern auch die Barrieren „in Form von Haltungen, Stimmungen, Atmosphären, also Begegnungs- und Verstehenskulturen" in den Blick nehmen. ${ }^{15}$ Paul Mecheril plädiert für eine selbstreflexive Praxis, die kritisch die bestehenden Differenzordnungen der Gesellschaft anfragt. Ausschließende Verhältnisse könnten nicht „einfach“ durch eine inklusive Pädagogik überwunden werden. Durch kritisches Wahrnehmen und die Reflexion handlungsprägender Unterscheidungsideologien können pädagogisch Arbeitende aber anfangen, Ausschlüsse wahrzunehmen und kontinuierlich nach Wegen der Inklusion zu suchen - ohne den Anspruch darauf zu erheben, Inklusion verwirklicht zu haben. ${ }^{16}$ Zum anderen sollten Lehrende ihre eigene lebensweltliche Verortung reflektieren und „den eigenen kultur- und milieuspezifischen Blick auf Lernende und ihre Lebenswelten [...] schärfen“, um „heterogenitätssensible Bildungsprozesse erforschen und initiieren zu können "17. Hierzu gehört eine Sensibilisierung dafür, nicht das eigene Milieu als „normal“ vorauszusetzen und „damit implizit die Lebenswelten der Schülerinnen und Schüler als anders, fremd - und auch als ,nicht-normal' zu qualifizieren ${ }^{\text {"18 }}$. Religionspädagogen sollten, so Gärtner, dazu befähigt werden, „die Heterogenität von Lebenswelten“ wahrzunehmen und zwar auch hinsichtlich religiöser Verschiedenheit. ${ }^{19}$ Dabei sollten auch Einschließungsund Ausgrenzungspraktiken aufgrund von Kultur und Religion zur Sprache kommen.

\footnotetext{
14 Flake/Schröder, Pädagogik, 42.

15 Markus Dederich, Behinderung als sozial- und kulturwissenschaftliche Kategorie, in: Markus Dederich/Wolfgang Jantzen (Hg.), Behinderung und Anerkennung, Band 2, Stuttgart 2009, 15-40, 17.

16 Vgl. Paul Mecheril, Einführung in die Migrationspädagogik, Weinheim/Basel 2004, 221-224.

17 Claudia Gärtner, Konfessioneller Religionsunterricht - kulturelle Vielfalt als Herausforderung eines auf Homogenität konzipierten und auf Identität zielenden Faches, in: Franz Gmainer-Pranzl et al. (Hg.), Herausforderung Interkultureller Theologie, Beiträge zur Komparativen Theologie 26, Paderborn 2016, 89-104, 102.

18 Ebd.

19 Ebd.
} 


\section{Religiöses Diversitätsbewusstsein und „Religiöses Othering“ (P. Mecheril/O. Thomas-Olalde)}

Paul Mecheril und Oscar Thomas-Olalde zeigen eine Disparität des gegenwärtigen Diskurses über Religion und religiöse Pluralisierung auf. Auf der einen Seite finden sich Ansätze, die Religion „in komplexen, individualisierten, pluralen und globalisierten Gesellschaften als fluides, optionales und deinstitutionalisiertes Phänomen" betrachten, auf der anderen Seite wird auf die lebensweltliche Relevanz von Religion und ihre Wirkmächtigkeit als „subjektivierende, kulturelle, gesellschaftliche und politische" Kraft hingewiesen. ${ }^{20} \mathrm{Nach}$ Mecheril und Thomas-Olalde fungiert Religion als „natio-ethno-kultureller Zugehörigkeitscode“21. Sie ist eine „soziale Praxis, ein Kommunikations- und Imaginationsschema“, welche(s) zur Unterscheidung von Gruppen genutzt werden kann. ${ }^{22}$ Dabei, so Mecheril und Thomas-Olalde, bringen Diskurse und Praxen Subjekte hervor und bestimmen deren Positionierung in der Gesellschaft. Julia Reuter übersetzt „Othering“ treffend mit „VerAnderung“, um diese Fremdheit produzierende Praktik auf einen Begriff zu bringen. ${ }^{23}$ Die eigene Angst und Unsicherheit wird auf den Anderen projiziert, um Macht und Autorität zu etablieren und aufrechtzuerhalten und die eigene „Normalität“ zu bestätigen. ${ }^{24}$ Durch diese Positionierung und das ,religiöse Othering ${ }^{c}$ werden „politische und gesellschaftliche Praktiken schliesslich [!] ermöglicht, legitimiert oder in Frage“ "gestellt. ${ }^{25}$,Religiöses Othering' finde nicht ohne Grund im Kontext von Globalisierung und Migration statt. Religion werde dabei „mit Andersheit (Otherness) verbunden“26. Die „andere Religion“ sei „in diesem diskursiven Topoi immer die „Religion der Anderen“, womit die Andersheit der Anderen als quasi-religiöse Setzung festgelegt" werde. ${ }^{27}$ Nicht ganz zufällig sei man bei der Suche nach einer Legitimation für die Behandlung des Einwanderers als „Fremden“ auf die Religion gestoßen: „Der religiöse Blick

20 Paul Mecheril/Oscar Thomas-Olalde, Die Religion der Anderen. Anmerkungen zu Subjektivierungspraxen der Gegenwart, in: Brigit Allenbach et AL. (Hg.), Jugend, Migration und Religion. Interdisziplinäre Perspektiven, Religion - Wirtschaft - Politik 4, Zürich 2011, 35-66, 44-45.

21 A.a.O., 57.

22 A.a.O., 58.

23 Julia Reuter, Ordnungen des Anderen. Zum Problem des Eigenen in der Soziologie des Fremden, Bielefeld 2002, 148. Vgl. auch Claudia JAHNEL, Interkulturelle Theologie und Kulturwissenschaft. Untersucht am Beispiel afrikanischer Theologie, Stuttgart 2016, 36 .

24 Vgl. Kim, Politik, 154.

25 A.a.O., 37.

26 A.a.O., 46.

27 Ebd. 
eröffnet“, so Mecheril und Thomas-Olalde, „eine einmalige Möglichkeit, den als Nicht-EU-Bürger verbliebenen ,Einwanderer-Resten ein eindeutiges gemeinsames Merkmal zuzuschreiben, nämlich eine orientalisch-islamische Grundeinstellung. “28 Hierbei werden ,die Anderen` zum Kontrastschemata, um ein einheitliches „Wir“ zu konstituieren, dem „überwiegend positive Eigenschaften" zugeschrieben werden. ${ }^{29}$ Angesichts der als Bedrohung wahrgenommenen Vielfältigkeit kann somit ein Einigkeitsempfinden geschaffen werden. Dieses „Wir-Gefühl“, das durch die Konstruktion des Fremden durch religiöses Othering gewonnen wird, ist jedoch nicht stabil, weil es die religiöskulturellen Zwischenräume negieren muss und so der stetigen Erneuerung durch Fremdzuschreibungen bedarf. Es blendet aus, wie verwoben Islam, Christentum und Judentum „in historischer, gesellschaftlicher, kultureller, sozialer, theologischer und anderer Perspektive“30 sind. Für Individuen und Gruppen hat das ,religiöse Othering gravierende Auswirkungen, weil die dadurch bestimmten Subjektivierungspraxen „alltägliche, lebensweltliche Konsequenzen" haben und über Zugang zu und Teilhabe an Ressourcen entscheiden. ${ }^{31}$

Mecheril und Thomas-Olalde formulieren ihren Begriff des „religiösen Otherings“ in Bezug auf Saids Orientalismuskritik. Betrachtet man "Othering“ als Prozess oder Vorgang, bei dem andere Menschen oder Gruppen als „fremd“ klassifiziert werden, um sich selbst hervorzuheben und eine Vormachtstellung zu behaupten, dann kann der Begriff des religiösen Otherings auch aufmerksam machen für intrareligiöse Dominanzprozesse bzw. für ein intrareligiöses Othering innerhalb des Christentums. So werden Beiträge aus der kontextuellen Theologie oft immer noch nicht als solche behandelt, die grundsätzlich etwas zur Theologie beitragen können und umgekehrt die westliche Theologie nicht als kontextuelle Theologie gesehen, sondern als universal gültig. Auch das intrareligiöse Othering hat lebensweltliche Konsequenzen, weil es über Zugang zu und Teilhabe an Ressourcen entscheidet und kontextuelle Theologie in der Sprache der vorherrschenden Theologie formuliert werden muss, um Gehör zu finden. So beschreibt beispielsweise Musa Wenkosi Dube, Bibelwissenschaftlerin in Botswana, dass sie ihr Studium in Großbritannien und den USA absolvierte und dort "das Gefühl dafür bekam, wie stark verwestlicht Theologie und Exegese sind und wie kolonisierend es sich anfühlte in solch

\footnotetext{
28 Ebd.

29 A.a.O., 53.62.

30 Iman Attia/Ozan Keskinkilic, Antimuslimischer Rassismus, in: Paul Mecheril (Hg.) unter Mitarbeit von Veronika Kowabas und Matthias Rangger, Handbuch Migrationspädagogik, Weinheim/Basel 2016, 168-182, 169 .

31 A.a.O., 49 .
} 
einem Postgraduierten-Programm zu sein", in dem die Studienprogramme und deren Literatur sehr ausschließend wirkten. Dube plädiert stattdessen für eine Wissensproduktion, die „wirklich divers und repräsentativ ist für die Welt, wie sie ist - in all ihren Diversitäten“, für eine Theologie, „die nicht so eng von einer einzigen Perspektive her definiert ist “32.

Will schulische Bildung Mündigkeit, Demokratie und Alteritätskompetenz stärken, sollten Schüler deshalb auch zur Wahrnehmung „religiösen Otherings“ und "intrareligiösen Otherings" sensibilisiert werden. Im Folgenden möchte ich deshalb genauer entfalten, dass es in vielerlei Hinsicht notwendig und bereichernd ist, die intrareligiöse Pluralität wahrzunehmen und Interkulturelle Theologie zum Bestandteil des Religionsunterrichtes zu machen.

Interkulturelle Theologie als herausfordernde Ressource

Interkulturelle Theologie „erkundet die interkonfessionellen, interkulturellen und interreligiösen Dimensionen des christlichen Glaubens“33. Infolge des Globalisierungsschubes hat sich nach Volker Küster eine Epochenwende in der Interkulturellen Theologie vollzogen: Einerseits ist hierdurch ein ständiges theologisches „Verhandeln zwischen dem Lokalen und dem Globalen notwendig, ein Prozess, der durch Ambiguität und Fluidität gekennzeichnet ist “34. Andererseits kommt es zunehmend zu einer Verschmelzung kontextueller und Interkultureller Theologie. ${ }^{35}$ Den Begriff „Interkulturelle Theologie“ verwende ich im Folgenden nicht als Disziplinenbegriff, sondern im Sinne einer bestimmten "Art des Theologietreibens", einer Perspektive im und für das Theologisieren: ${ }^{36}$ Interkulturelle Theologie konfrontiert mit "kontrastierenden Wahrheitsansprüchen“ und pluralen, aus der Sicht westlicher Christen „differenten Bezeugungen und Formulierungen christlicher Identität“ ${ }^{37}$ Sie bringt zum Bewusstsein, dass, wie Estermann es formuliert, Gott nicht europäisch

32 Bernhard Offenberger, „To push the boundaries“. Die Grenzen des Wissens weiten. Ein Interview mit Musa W. Dube, in: BiKi 67 (2012), 160-163, 161.

33 Volker Küster, Einführung in die Interkulturelle Theologie, Göttingen 2011, Klappentext.

34 A.a.O., 27.

35 Ebd.

36 Ulrich Dehn, Die Seite der Schriftleitung, in: Interkulturelle Theologie 40 (2014), 122$124,122$.

37 Judith Gruber, Theologie nach dem Cultural Turn. Interkulturalität als theologische Ressource, ReligionsKulturen 12, Stuttgart 2013, 37-38. 
ist und die Theologie nicht abendländisch. ${ }^{38}$ Seit Ende der 6oer Jahre des 20. Jahrhunderts gibt es in allen Ländern des Trikont kontextuelle Theologien, die einen epistemologischen Bruch mit der akademischen Theologie des Westens proklamieren. ${ }^{39}$ SoentwickeltesichinLateinamerikaeine „Befreiungstheologie“, in Südkorea eine „Minjung-Theologie“, in Nordamerika und Südafrika gibt es die „Schwarze Theologie“. Neben den Befreiungstheologien gibt es in Afrika und Asien christliche „Inkulturations- und Dialogtheologien “40. Während die Inkulturationstheologien dem christlichen Glauben eine einheimische Gestalt geben und teilweise auch Elemente anderer Religionen integrieren, suchen Dialogtheologien das interreligiöse Gespräch. ${ }^{41}$ Auch wenn viele kontextuelle Theologien immer noch nicht Eingang in die theologischen und schulischen Curricula (auch ihrer Herkunftsländer) gefunden haben ${ }^{42}$ sind sie inzwischen $\mathrm{zu}$ "globalen theologischen Strömungen" angewachsen. ${ }^{43}$

Orlando O. Espín formuliert aus der Sicht US-amerikanischer Latinos und Latinas zwei grundlegende Einsichten der Interkulturellen Theologie: ${ }^{44}$ Alle Theologen sollten erkennen, von wo aus und von wem aus sie Theologie betreiben. Theologie ist damit "ein begrenztes, provinzielles Produkt" und nicht „neutral“. ${ }^{45}$ Die Wahrheit ist deshalb nach Espín „ein kultureller und ein interkultureller Prozess" ${ }^{46}$.

$\mathrm{Zu}$ diesem kulturellen und interkulturellen Suchprozess kann der Religionsunterricht ermutigen. Interkulturelle Theologie ist, so die These, die ich im Folgenden darlegen möchte, in zweierlei Hinsicht eine herausfordernde Ressource für den Religionsunterricht im Zeitalter von Migration und Globalisierung: Zum einen ist sie eine herausfordernde Ressource für das Konfessionsverständnis im konfessionellen Religionsunterricht. Sie fordert

38 Josef Estermann, Gott ist nicht europäisch, und die Theologie ist nicht abendländisch. Eine interkulturelle Dekonstruktion der abendländischen Theologie aufgrund der indigenen andinen Weisheit, in: IKTh 40 (2014), 186-200.

39 KÜSTER, Einführung, 55 .

40 A.a.O., 57.

41 Ebd.

42 Ebd.

43 A.a.O., 89.

44 Orlando O. Espín, Die Theologie der Latinos/Latinas in den USA als Experiment in der Interkulturellen Theologie, in: Wolfgang Gantke/Thomas Schreijäck (Hg.), Religionen im Kulturwandel zwischen Selbstannahme und Selbstaufgabe. Kontinentalkontextuelle Perspektiven, Forum Religionspädagogik Interkulturell 21, Berlin 2011, 33-48, $35^{-} 36$.

45 ЕАтшот, Abschlusserklärung der Konferenz von Daressalam 1976, in: Von Gott reden im Kontext der Armut. Dokumente der Ökumenischen Vereinigung von Dritte-WeltTheologinnen und -Theologen 1976-1996, Freiburg i.Br. 1999, 33-46, 44.

46 Espín, Theologie, $35^{-36}$. 
dazu heraus, ein konfessionelles Selbstverständnis zu entwickeln, das nicht auf einem ,religiösen Othering' und auch nicht auf intrareligiösem Othering basiert. Die Forderung nach Inklusion im Kontext kultureller und religiöser Pluralisierung ist im gegenwärtigen konfessionellen Religionsunterricht nur umsetzbar, wenn die Realität christlicher Binnendifferenzierung und die kulturelle Vielfalt des Christentums umfassend reflektiert werden.

Zum anderen ist Interkulturelle Theologie eine herausfordernde Ressource für das Theologisieren mit Schülerinnen und Schülern. Sie konfrontiert mit kontrastierenden Wahrheitsansprüchen anderer Glaubensvorstellungen und wird dadurch zur Herausforderung, aber auch zur Ressource für die religiöse Identitätsbildung im Kontext von Migration und Globalisierung. Sie kann für Prozesse des ,religiösen Otherings' sensibilisieren und eine Brücke zum interreligiösen Lernen bilden, weil sie zur Konstituierung eines heterogenitätssensiblen „Ichs" und "Wirs" ermutigt.

Interkulturelle Theologie als herausfordernde Ressource für das Konfessionsverständnis im konfessionellen Religionsunterricht

Derzeit sind in der Religionspädagogik zwei Reaktionen auf die kulturelle und religiöse Pluralisierung erkennbar, die sich gegenseitig nicht ausschließen: Zum einen lässt sich in Forschung, Lehre und RU ein breites Interesse an Interreligiöser Bildung und Kommunikation ausmachen. Zum anderen gibt es eine breite Befürwortung des konfessionellen Religionsunterrichtes (und des konfessionell-kooperativen Religionsunterrichtes).

In einem Positionspapier von 163 Religionspädagoginnen und Religionspädagogen aus Forschung und Lehre wurde 2016 eine Weichenstellung für die Ausrichtung des Religionsunterrichtes vorgenommen. Anhand der Leitbegriffe „konfessionell“, „kooperativ“ und „kontextuell“ werden Vorschläge für die zukünftige Gestaltung des Religionsunterrichtes dargelegt. ${ }^{47}$ Das Positionspapier spricht sich für den konfessionellen und für den konfessionellkooperativen Religionsunterricht aus. Schülerinnen und Schüler sollen hier „verstehen lernen, wie Christsein angesichts heutiger Lebensbedingungen möglich ist“48. Es weist dabei darauf hin, dass „das Christentum in Konfessionen

47 Positionspapier: Damit der Religionsunterricht in Deutschland zukunftsfähig bleibt. „Konfessionell, kooperativ, kontextuell - Weichenstellungen für einen zukunftsfähigen Religionsunterricht“ http://akrk.eu/positionspapier-damit-der-religionsunterrichtin-deutschland-zukunftsfaehig-bleibt/.

Ebd. 
ausgeprägt ist und selbst individuelle Bezüge auf das Christentum - bewusst oder unbewusst - konfessionelle Akzentsetzungen aufgreifen ${ }^{49}$. Dabei gehe es darum, die Vielfalt des Christlichen als Reichtum wachzuhalten, ohne in traditionelle Muster der Konfessionalisierung zu verfallen. ${ }^{50}$ Für die Ausgestaltung des zukünftigen konfessionellen RUs seien religionsdidaktische Eckpunkte und Standards zu formulieren und zu etablieren. ${ }^{51}$

Dieser Hinweis ist m.E. von besonderer Bedeutung, da der konfessionelle Religionsunterricht bisher ein Fach ist, das, wie Claudia Gärtner aufzeigt, „auf Homogenität konzipiert ist und auf Identität zielt“52. Auch Ulrike LinkWieczorek weist darauf hin, dass die Vorstellung noch immer verbreitet sei, dass sich im Prozess der religiösen Sozialisation zuerst eine konfessionelle Identität ausprägt, die dann in den Diskurs mit anderen Identitäten (Konfessionen oder Religionen) eintreten könne. Implizit werde dabei, so Link-Wieczorek, eine Homogenität religiöser Sozialisationsprozesse vorausgesetzt, wie sie auch in einem Deutschland jenseits aller Migrationseinflüsse kaum noch anzutreffen sei. Link-Wieczorek plädiert dagegen dafür, „die Einsichten in den Zusammenhang von Identität und Dialog ernst zu nehmen, derzufolge [sic] Menschen in pluralen Gesellschaften ihre religiöse Identität in ihrer jeweiligen Lebenswelt in Auseinandersetzung und Begegnung formen“, was auch für die konfessionelle Identität gelte. ${ }^{53}$ Vor allem muss nach Link-Wieczorek die zunehmende Binnendifferenzierung der Konfessionen ernst genommen werden. Religiös-kulturelle Differenzen bestehen nicht nur zwischen den Religionen, sondern laufen "mitten durch sie hindurch“, auch „mitten durch das Christentum “54. „Die Zeiten, in denen die Ökumene vornehmlich mit der Differenz zwischen evangelisch und katholisch verbunden wurde, gehen“, so Link-Wieczorek „endgültig ihrem Ende entgegen.“55 Die Umwandlung der europäischen Gesellschaften in Migrationsgesellschaften werde aufmerksamer machen für die Realität christlicher Binnendifferenzierung. So handele es sich bei syrischen Flüchtlingen beispielsweise „zu einem großen Teil um Christinnen und Christen“, „die uns zunächst einmal kaum weniger fremd sind als die muslimischen bosnischen Flüchtlinge zur Zeit des Jugoslawienkonfliktes“56.

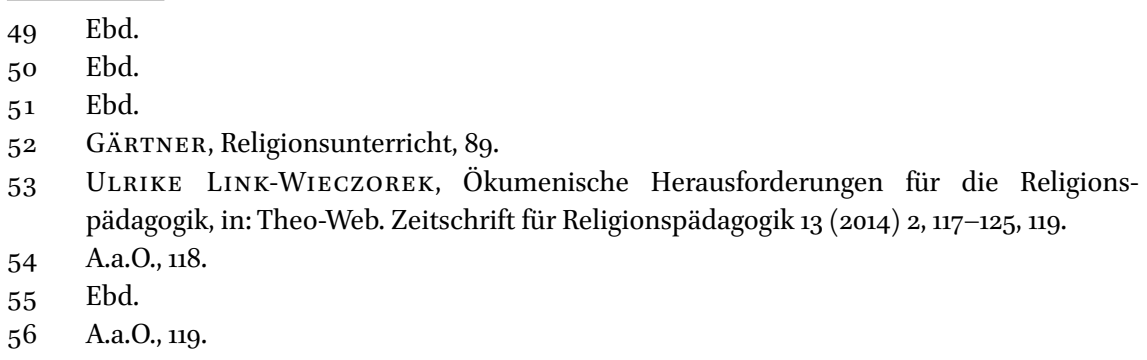


Dennoch, so Link-Wieczorek, erhalten wir „durch sie in spezifischer Weise einen neuen Blick auf unsere ,eigene“ religiöse Tradition“57.

Mit Claudia Gärtner bin ich der Ansicht, dass der konfessionelle Religionsunterricht diese gesellschaftliche und intrareligiöse Pluralität bisher "nicht umfassend genug reflektiert" ${ }^{458}$. Dabei ist es eine dringende Herausforderung der Gegenwart, eine christliche Identität auszubilden, die nicht in "traditionelle Muster der Konfessionalisierung “ verfällt, sondern die religiöse Vielfalt und auch die intrareligiöse Pluralität zur Entfaltung bringt. Will man der Gefahr entgehen, im konfessionellen Religionsunterricht in „traditionelle Muster der Konfessionalisierung " zu verfallen oder auf eine Konzeption des „Wir“ (Christen) und „die Anderen“ (Religionen) zurückzugreifen, steht zu Beginn die Akzeptanz der Vielfältigkeit von Glaubensansichten innerhalb des Christentums, aber auch die Akzeptanz der kulturellen Pluralität der Bibel. Dann kann das Potential erkannt werden, das in einer bewusst interkulturell angelegten Hermeneutik der Bibel liegt. ${ }^{59}$ „Wer sich mit der Bibel beschäftigt", so Rita Müller-Fieberg, „begibt sich unweigerlich auf interkulturelles Terrain. “60 Die Bibel ist, so fährt sie fort, „ein Zeugnis der Begegnung verschiedener Religionen, Kulturen und Sprachen“61. Christliche Identität wird „von Anfang an im interkulturellen Raum verhandelt und besteht in einer Vielzahl an Christentümern“62. Auch die Exegese war und ist deshalb „immer schon eine Disziplin, die unter dem Vorzeichen der Interkulturalität steht ${ }^{463}$.

Es ist deshalb notwendig, sich von einer eurozentristischen Deutungshoheit zu verabschieden und sich auf die "globale Erzähl- und Interpretationsgemeinschaft der Christenheit“ einzulassen. ${ }^{64}$ Dazu gehört auch, in Betracht zu ziehen, dass "Christinnen und Christen außereuropäischer Herkunft sozialgeschichtlich wie vom Weltbild her manchmal der antiken mediterranen Welt vielleicht sogar näher stehen als mitteleuropäische Rezipienten, deren Perspektive durch die individualisierende

\section{Ebd.}

58 GÄRTNER, Religionsunterricht, 91.

59 Rita Müller-Fieberg, Interkulturelle Lernerfahrungen mit der Bibel, in: Franz Gmainer-Pranzl et AL. (Hg.), Herausforderung Interkultureller Theologie, Beiträge zur Komparativen Theologie 26, Paderborn 2016, 67-85, 75 .

6 A.a.O., 74 .

61 A.a.O., 75 .

62 Gruber, Theologie, 75.

63 MÜLlER-FIEBERG, Lernerfahrungen, 76.

64 Volker Küster, Von fremden Christologien lernen. Das Antlitz Jesu Christi in Afrika, Asien und Lateinamerika neu entdecken, in: Helmut A. Müller (Hg.), Kultur, Religion und Glauben neu denken. Von der abrahamitischen Ökumene zur Ökumene der Weltreligionen, Berlin 2014, 65-84, 84 . 
Aufklärungstradition geprägt ist"65. So ist, wie Michael Nausner zu Recht aufzeigt, beispielsweise die Erfahrung von Migration und einer gewissen existentiellen Heimatlosigkeit ein so durchgängiges Thema in der Bibel, dass man sich fragen könne, ob aus christlicher Perspektive der epistemologische Blick der Migrierenden teilweise nicht ein schärferer sein müsste als derjenige der sesshaften christlichen Gemeinschaften. ${ }^{66}$ Auch die von uns in Deutschland als "objektiv“ oder "neutral“ wahrgenommene Exegese ist, wie Werner Kahl zu recht anmerkt, „eine Spielart kontextueller Exegese“ ${ }^{“ 67}$.

Von hier aus weitergedacht ergibt sich die Frage, wie Interkulturelle Theologie religiöse Bildungsprozesse im Konfessionellen und Konfessionellkooperativen Religionsunterricht bereichern, aber auch herausfordern kann.

\section{$6 \quad$ Interkulturelle Theologie als herausfordernde Ressource für das Theologisieren}

Interkulturelle Theologie, so lautet hieran anschließend meine zweite These, ist eine herausfordernde Ressource für das Theologisieren mit Schülerinnen und Schülern. Sie konfrontiert mit kontrastierenden Wahrheitsansprüchen anderer Glaubensvorstellungen innerhalb des Christentums und wird dadurch zur Herausforderung, aber auch zur Ressource für die religiöse Identitätsbildung. Sie kann eine Brücke zum interreligiösen Lernen bilden.

Beim Theologisieren werden Schülerinnen und Schülerals Gesprächspartner und eigenständige Konstrukteure des Wissens ernstgenommen, indem sie motiviert werden, eigene Fragen und Antworten zu finden. ${ }^{68}$ Das Theologisieren ist ein „Reflexionsverhältnis“ über sich selbst und die eigenen theologischen Vorstellungen. ${ }^{69}$ Die Lehrenden helfen, Gedanken zu formulieren, zu vertiefen und ermutigen "selbst theologisch nachzudenken" ${ }^{40}$. Sie schulen die theologischeUrteilsfähigkeitund „denAufbauderdafürerforderlichenFähigkeitenund Kompetenzen“71. Die Auseinandersetzung mit grundsätzlichen theologischen

65 So Müller-Fieberg im Rekurs auf Werner Kahl, Dies., Lernerfahrungen, 74.

66 Michael NaUsner, Wer darf dazugehören? Anstöße Postkolonialer Theologie im Kontext der Migration, in diesem Band, 40.

67 Werner KAHL, Westafrikanische Bibelinterpretationen. Irritationen, Herausforderungen, Bereicherungen, in: BiKi 67 (2012), 149-153, 151.

68 Vgl. Friedrich Schweitzer/Thomas Schlag, Brauchen Jugendliche Theologie? Jugendtheologie als Herausforderung und didaktische Perspektive, Neukirchen-Vluyn 2011, 28.

69 Ebd.

70 A.a.O., $28-29$.

71 Ebd. 
Fragen soll Schülerinnen und Schülern helfen, Orientierung zu gewinnen und weiterführende Deutungs- und Orientierungsangebote kennenzulernen. Religiöse Identitätsbildung ist hierbei als ein Prozess zu verstehen, bei dem „in der Wahrnehmung und Interaktion von verschiedenen Lebenskonzepten und Selbst- und Weltdeutungen ein eigenes ,Identitätsgewebe“" entsteht. Dieses ist nicht statisch, sondern kann sich „lebenslang verändern oder verstärken, zerreißen oder verdichten" ${ }^{\prime 72}$.

Freudenberger-Lötz macht zwei Richtungen des Theologisierens aus: Zum einen sollen Schülerinnen und Schüler zu Spurensuchern von "gelebter Religion" gemacht werden. ${ }^{73}$ Zum anderen sollten Lehrende mit den Schülern „bedeutungsvollen Spuren in der biblischen Überlieferung bzw. christlichen Tradition" nachgehen. ${ }^{74}$ Wichtig ist, dass die Lehrenden Angebote zum Weiterdenken machen. Sie sollten "gewagte Hypothesen“ einbringen, die Schülerinnen und Schüler zu eigenen Antworten herausfordern und ihnen Ordnungs- und Orientierungshilfe bieten. ${ }^{75}$ Die "gewagten Hypothesen“ bilden sich nach Freudenberger-Lötz z.B. daraus, dass „eine bestimmte Erfahrung" an ein "Urthema des christlichen Glaubens" angebunden ist oder indem "eine bestimmte Glaubenserfahrung, die in satzhafter Form überliefert ist, eine moderne Lebenserfahrung trifft" ${ }^{\text {"76: }}$ Als ein solches Deutungsund Orientierungsangebot darf meiner Ansicht nach nicht nur traditionelle Theologie europäischer Provenienz eingebracht werden. Stattdessen müssten beispielsweise auch Christologien von Theologen und Theologinnen aus Afrika, Asien und Lateinamerika eingebracht werden. Dabei ist es auch notwendig, zu reflektieren, in welchen Medien alternative Theologien zur Sprache gebracht, und auch Lieder, Gedichte, Bilder, Theater und Film zur Kenntnis genommen werden. ${ }^{77}$ Spannend wäre für schulische Prozesse zudem, Austauschprozesse anzuregen, bei denen Schülerinnen und Schüler direkt mit christlichen

72 LARS Wolf, Ökumenischer Religionsunterricht - ein Beitrag zur Integration, in: PrTh 37 (2002), 16-22, 20.

73 Petra Freudenberger-Lötz, Theologische Gespräche mit Kindern, Erfahrungen Beispiele - Anleitungen. Ein Werkstattbuch für die Sekundarstufe, München 2012, 49.

74 Ebd.

75 A.a.O., 50. Petra Freudenberger-Lötz folgt hier dem in Anlehnung an Ulrich Oevermann formulierten Konzept der „abduktiven Korrelation“: Durch das Einbringen "gewagter Hypothesen" sollen Diskurshorizonte eröffnet werden. Hans Georg Ziebertz et AL., Abduktive Korrelation, 27. Vgl. hierzu: Petra Freudenberger-Lötz, Theologische Gespräche mit Kindern, 51 .

76 Petra Freudenberger-Lötz, Theologische Gespräche mit Kindern, Erfahrungen Beispiele - Anleitungen. Ein Werkstattbuch für die Sekundarstufe, München 2012, 51.

77 Doris Bachmann-Medick, Cultural turns. Neuorientierungen in den Kulturwissenschaften, Hamburg $5_{2014}, 191$. 
Schülerinnen und Schülern anderer Länder über Glaubensvorstellungen kommunizieren. Hierbei könnten auch Vernetzungsmöglichkeiten über das Internet genutzt werden.

Theologisieren im Dialog mit Interkultureller Theologie kann dann dafür sensibilisieren, dass die Konstituierung einer bestimmten religiösen „Identität“ zunächst ein kultureller Sozialisierungsprozess ist: „Die konkrete, uns wie eine zweite Haut umgebende Kultur (oder Un-Kultur) vermittelt - ob wir es wollen oder nicht - religiöse Vorstellungen und diesen zugrundeliegenden Denkschemata“, so Josef Estermann. ${ }^{78}$ Interkulturelle Theologie stellt hierbei eine Herausforderung dar, eine Standortbestimmung zu vollziehen und ggf. den eigenen, privilegierten Status und eigene stereotype Denkweisen zu hinterfragen. Sie fordert ein, die intrareligiöse Pluralität und Vielfältigkeit christlicher Glaubensvorstellungen zur Kenntnis zu nehmen.

Diese Denkprozesse setzen ein komplexes und differenziertes Selbstreflexionsvermögen voraus, das nicht bei allen Schülerinnen und Schülern vorhanden ist. Deshalb ist es notwendig, durch Prozesse des Theologisierens eine reflexive Haltung anzubahnen und dabei immer wieder auch "Fremdheitserfahrungen“ zu thematisieren. Ricœur weist darauf hin, dass die Entdeckung des kulturellen Pluralismus keine "harmlose Spielerei“ ist: „In dem Augenblick, wo wir entdecken, daß es Kulturen und nicht nur eine Kultur gibt, in dem Augenblick also, wo wir das Ende eines illusorischen oder realen kulturellen Monopols zugeben, in diesem Augenblick bedroht unsere eigene Entdeckung uns mit zerstörerischer Kraft. Plötzlich schält sich die Möglichkeit heraus, daß es nur noch die anderen gibt, daß wir selbst ein anderer unter anderen sind. ${ }^{49}$ Wichtig ist dabei die Erkenntnis, dass der Prozess der Einübung in das Verstehen des „Fremden“ dabei beginnt, eigene innere Widersprüchlichkeiten zu entdecken und sich selbst teilweise auch als "fremd“ und „fremd bestimmt, zu erkennen. ${ }^{80}$ In diesem Prozess kann dann ein neues Selbstverständnis entstehen, „das weiter und weltweit anschlussfähiger ist als die Konzeption des autonomen europäischen Individuums ${ }^{\text {"81. }}$. Sehr erhellend sind hierfür die Ausführungen von Michael Nausner, die er in diesem Aufsatzband im Anschluss an Bernhard Waldenfels formuliert. Nach

78 Josef Estermann, Schritte zu einem polyzentrischen Religionsbegriff. Herausforderungen für die Religionspädagogik aus der Perspektive der interkulturellen philosophischen Diskussion, in: Thomas SchreıjÄck (Hg.), Christwerden im Kulturwandel. Analysen, Themen und Optionen für Religionspädagogik und Praktische Theologie. Ein Handbuch, Freiburg i.Br. 2001, 200-223, 202-203.

79 Paul Ricceur, Geschichte und Wahrheit, München 1974, 285.

8o BaChMANN-Medick, turns, 206.

81 Ebd. 
Waldenfels beginnt „die Fremdheit“ in mir, „im eigenen Hause“82, er zeigt auf, dass "niemand sich je selbst ganz gehört" und niemand seiner Kultur in Gänze zugehörig ist. ${ }^{83}$ Diese grundlegende innere Fremdheit thematisiert exemplarisch der Song „Das ist dein Leben“ von Philipp Dittberner, der im September 2015 erschien und Platz 12 in den deutschen Albumcharts erreichte:

Das ist dein Leben, das ist, wie du lebst.

Warum du liebst und lachst und dich selbst nicht so verstehst. Warum du dir wieder so fremd bist, in einer doch so hellen Zeit. Warum du den wieder vermisst, der dich sicher nicht befreit. Ja genau, das ist dein Leben; das ist, wie du lebst.

Warum wir manchmal fliegen; nicht mal wissen, wie es geht. Und wir immer wieder aufstehen und anfangen zu gehen.

Ja genau, das ist dein Leben, und du wirst es nie verstehen.

Nausner folgert aus der These einer grundlegenden inneren Fremdheit, dass „die Einbeziehung verschiedener Perspektiven nicht ein Akt der Großzügigkeit [ist], sondern eine Bedingung für ein angemessenes Verständnis kultureller Komplexität ${ }^{\text {u44 }}$. Erst wenn man die eigene Zugehörigkeit als abhängig von anderen verstehe, könne man sich selbst verstehen. Von dieser Selbsterkenntnis ausgehend, dass ich mich selbst manchmal nicht verstehe und mir selbst immer wieder neu „auf die Spur kommen“ muss, kann die Fremdheit des Anderen als Ressource und Herausforderung und nicht als Bedrohung des Selbst wahrgenommen werden, wodurch sich, wie Nausner in Bezug auf Bernhard Waldenfels sagt, „Wege zur Fremdheit des Anderen“ eröffnen. ${ }^{85}$

Theologisieren als Suche nach kulturell-religiösen Zwischenräumen

Interkulturelle Theologie wird zur Ressource für das Theologisieren, wenn sie Schülerinnen und Schüler zu Spurensuchern von kulturell-religiösen

\footnotetext{
82 Bernhard Waldenfels, Grundmotive einer Phänomenologie des Fremden, Frankfurt am Main 2006, 120.

83 A.a.O., $118-120$.

84 Michael Nausner, Wer darf dazugehören? Anstöße Postkolonialer Theologie im Kontext der Migration, in diesem Band, 34.

85 Michael Nausner, Wer darf dazugehören? Anstöße Postkolonialer Theologie im Kontext der Migration, in diesem Band, 26. WALDEnfELs, Grundmotive, 84.
} 
Zwischenräumen und Kontaktzonen macht. Schülerinnen und Schüler können dabei erkennen, dass der größte Teil der Menschheit „in Kontaktzonen ansässig“ ist, wo „verschiedene Kulturen zusammentreffen, aufeinanderstoßen und sich auseinandersetzen". ${ }^{86}$ Sie können lernen, dass sich jedoch auch viele Menschen in einem „gewaltsamen kulturellen "Dazwischen-Sein“ befinden und durch „Othering" entschieden wird, wer als "einer von uns“ gilt oder als „Fremder". 87

Für die Analyse dieser Prozesse des „Otherings“, bei denen willkürlich ein Merkmal unter Menschen herausgegriffen und als andersartig und "fremd“ klassifiziert wird, z.B. aufgrund von Geschlecht, Religionszugehörigkeit oder Herkunft, kann auf Erkenntnisse der postkolonialen Theorie zurückgegriffen werden. Das Ziel dieser Spurensuche von kulturell-religiösen Zwischenräumen ist, mit Homi Bhabha gedacht, kein identitätsstiftender kultureller Bedeutungskonsens. Es ist ein notwendigerweise bleibend unabgeschlossener Prozess des Aushandelns und der stetigen Neueinschreibung durch die Überschneidung verschiedener, oft widersprüchlicher Diskurse. Immer wieder neu müssen „konstruktive Überschneidungs- und Überlappungsphänomene ausfindig“ gemacht werden. ${ }^{88}$ Homi Bhabha hat für diesen „Schwellenraum zwischen den Identitätsbestimmungen“ 89 den Begriff „third space“ („Dritter Raum“) eingeführt. Hier wird, so Bhabha, ein Aufbrechen von Dominanzkulturen möglich durch kulturelle Hybridität, in der es einen Platz für Differenz ohne eine übernommene oder verordnete Hierarchie gibt. ${ }^{90}$ Veranschaulichen lässt sich dies beispielsweise mit der US-amerikanischen Bürgerrechtsbewegung, die der Gewalt und Repression ausgesetzt war, weil sich Schwarze gegen die Ausgrenzung wehrten, aber auch, weil es Weiße gab, die sich mit ihnen solidarisierten. Damit schufen weiße und schwarze Bürgerrechtler einen „third space“, für Martin Luther King die „beloved community“, in dem die übernommenen und verordneten Machtverhältnisse innerhalb der US-Gesellschaft in Frage gestellt wurden.

Homi Bhabha wird angefragt, ob er die Machtverhältnisse und sozialen und ökonomischen Ungleichheiten tief genug reflektiert und ob es tatsächlich möglich ist, einen hierarchiefreien ,third space“ zu schaffen. ${ }^{91}$ In ihrem berühmten Aufsatz „Can the Subaltern Speak?“ zeigt Gayatri C. Spivak auf, dass die

\footnotetext{
86 So KüSTER, Einführung, 17, im Rekurs auf Mary Louise Pratt.

87 Uriah Y. Kim, Die Politik des Othering in Nordamerika und im Buch der Richter, in: Conc(D) 49 (2013), 152-161, 154.

88 BACHMANN-MEDICK, turns, 199.

89 A.a.O., 205.

9o Номі К. Внавна, Die Verortung der Kultur, Stauffenburg Discussion 5, Tübingen 2007, 5 .

91 So z.B. BACHMANN-Medick, turns, 201.
} 
„Subalternen“, die Marginalisierten, ihre Rechte nicht in ihrer eigenen Sprache einklagen können. Sie sind, so Uriah Y. Kim, gezwungen, „in der Sprache der Herren dagegen zu protestieren, dass sie als das Andere konstruiert werden ${ }^{492}$. Die Intellektuellen müssen sich ihrer eigenen Verwicklung und der Gefahr der Aneignung des Anderen bewusst werden, so Spivak im Rekurs auf Derrida. Sie können nicht für die Subalternen sprechen. ${ }^{93}$ Aber auch wenn es schwierig ist, "für den Anderen zu sprechen, und die „eigene Rolle im Prozess des Othering genauer untersucht werden muss, dürfen die Intellektuellen nicht davon entbunden werden, dem Anderen beizustehen" 94 .

Im Sinne von Spivak sollte im Religionsunterricht die Gefahr der Aneignung des Anderen reflektiert und die Frage eines Sprechens für den Anderen problematisiert werden. Die Beschäftigung mit Interkultureller Theologie darf auch nicht der Gefahr erliegen, als „exotischer Bilderbogen“ missverstanden zu werden. ${ }^{95}$ Stattdessen sollte es, wie Volker Küster betont, „um das Einüben in interkulturelles Verstehen und Solidarität innerhalb der globalen Erzähl- und Interpretationsgemeinschaft der Christenheit" gehen. ${ }^{96}$ Dieses Einüben in das interkulturelle Verstehen und in Solidarität muss als bleibend unabgeschlossener Prozess gedacht und gestaltet werden. Im Zuge des Aushandelns und der Neueinschreibung durch die Überschneidung verschiedener und auch widersprüchlicher Diskurse können und müssen immer wieder Zwischenergebnisse ausgemacht werden, die jedoch fluide bleiben und bei Bedarf neu verhandelt und überschrieben werden. Wichtig ist für das Theologisieren dann, Schülerinnen und Schüler zu ermutigen, die Prozesshaftigkeit des Gespräches, die möglicherweise bleibenden Fragen und Widersprüchlichkeiten auszuhalten. Dabei sollte, wie Heinz Streib fordert, eine Art „Kultivierung von Fremdheit" entstehen.${ }^{97}$ Es stellt sich die Frage, ob das Gefühl der Fremdheit überhaupt grundsätzlich überwunden werden muss, ob sie nicht vielmehr elementar zum Leben gehört. ${ }^{98}$ Wichtig ist es letztlich aber, weder zu einer

92 KIM, Politik, 155 .

93 Gayatri Chakravorty Spivak, Can the Subaltern Speak? Postkolonialität und subalterne Artikulation (aus dem Englischen von Alexander Koskowicz und Stefan Nowotny), in: Boris Bude et Al. (Hg.), Es kommt darauf an, Texte zur Theorie der politischen Praxis 6, 18-118, 106.

94 KIM, Politik, 155.

95 KÜSTER, Einführung, 222.

96 Ebd.

97 Heinz Streib, Wie finden interreligiöse Lernprozesse bei Kindern und Jugendlichen statt? Skizze einer xenosophischen Religionsdidaktik, in: Peter Schreiner et AL. (Hg.), Handbuch interreligiöses Lernen, Gütersloh 2005, 230-243, 231.

98 Henning Wrogemann, Religionen im Gespräch. Ein Arbeitsbuch zum interreligiösen Lernen, Stuttgart 2008, 189 . 
Ablehnung vom Fremden als dem "ganz anderen“, noch zu einer Stilisierung oder aneignenden Deutung des Fremden zu führen..$^{99}$

Schülerinnen und Schüler können durch Interkulturelle Theologie befähigt werden zur Analyse der Konstruktionen von „Wir“ und den „Anderen“, auch in Bezug auf intrareligiöse Pluralität. Sie können lernen, sich von den Umbrüchen der globalisierten Welt in Frage stellen und in Anspruch nehmen zu lassen: ${ }^{100}$ Wie kann ich, wenn ich privilegiert bin, dem Anderen/ Marginalisierten beistehen? Wen kann ich in Anspruch nehmen, wenn ich meine Unrechtserfahrung selbst nicht zur Sprache bringen kann? Wenn ich bereit bin, eigene Normalitätsvorstellungen in Frage zu stellen, entdecke ich in der Interkulturellen Theologie Ressourcen zur Ausbildung einer Identität (auch Glaubensidentität), die nicht auf Abgrenzungsmustern basiert, sondern auf Solidarität. Interkulturelle Theologie zeigt, wie Glaube als eine Resonanzerfahrung in der konkreten Lebenswirklichkeit erfahren werden kann, die bei Menschen (auch in Deutschland) oft von ungewisser Lebensperspektive, von sozialer Not und Leid gekennzeichnet sind. Dadurch ergeben sich auch Anknüpfungspunkte für Schülerinnen und Schüler, die z.B. nicht aus einem gutsituierten, glücklichen Mittelschichtsmilieu kommen. Auch für nicht-glaubende Jugendliche können sich Anschlussmöglichkeiten über die ökonomische Nachhaltigkeitsdebatte ergeben, die von Theologen aus Ländern des Trikont geführt werden. ${ }^{101}$

Noch ist das "Othering“ eurozentrischen Denkens in die Theologie eingeschrieben - Interkulturelle Theologie fordert jedoch heraus, dieses Othering zu hinterfragen und die Vielfältigkeit der Gottesbilder, Jesusbilder und Menschenbilder des Christentums neu zu entdecken. In einem so verstandenen konfessionellen bzw. konfessionell-kooperativen Religionsunterricht kann das intrareligiöse Lernen anhand von Interkultureller Theologie die Fähigkeit schulen, sich seiner kontextgebundenen religiösen oder auch nichtreligiösen Vorstellungen bewusst zu werden und sich in einem Kontext „religiöser, kultureller und sozialer Vielfalt über religiöse Fragen auszutauschen und zu verständigen“, ohne dabei auf ausgrenzende und stereotypisierende Denkmuster zurückzugreifen. ${ }^{102}$ Intrareligiöses Lernen bildet damit eine

\footnotetext{
99 A.a.O., 187.

100 Vgl. Franz Gmainer-Pranzl, Der "Logos christlicher Hoffnung“ in globaler Verantwortung. Interkulturelle Theologie. Historische und hermeneutische Grundlagen, in: IKTh 40 (2014), 129-148, 139.

101 So z.B.: Meenyun Chun, Reis und Wasser. Eine feministische Theologie in Korea, Berlin 2012.

102 Positionspapier. Damit der Religionsunterricht in Deutschland zukunftsfähig bleibt.
} 
notwendige Brücke zum interreligiösen Lernen. Die Einsichten Interkultureller Theologie können fruchtbar gemacht werden für religiöse Bildungsprozesse.

\section{Bibliographie}

Affolderbach, Martin, Migration, Religion und Bildung - national und international, in: Peter Schreiner et AL. (Hg.), Handbuch Interreligiöses Lernen, Gütersloh 2005, 70-84.

Attia, Iman /Keskinkilic, Ozan, Antimuslimischer Rassismus, in: Paul Mecheril (Hg.) unter Mitarbeit von Veronika Kowabas und Matthias RANGGER, Handbuch Migrationspädagogik, Weinheim/Basel 2016, 168-182.

Bachmann-Medick, Doris, Cultural turns. Neuorientierungen in den Kulturwissenschaften, Hamburg ${ }^{5} 2014$.

Внавна, Номі K., Die Verortung der Kultur, Stauffenburg Discussion 5, Tübingen 2007.

Chun, Meenyun, Reis und Wasser. Eine feministische Theologie in Korea, Berlin 2012.

Dederich, MARKus, Behinderung als sozial- und kulturwissenschaftliche Kategorie, in: Markus Dederich/Wolfgang Jantzen (Hg.), Behinderung und Anerkennung, Band 2, Stuttgart 2009, 15-40.

Dehn, UlRich, Die Seite der Schriftleitung, in: IKTh 40 (2014), 122-124.

ЕАтшот, Abschlusserklärung der Konferenz von Daressalam 1976, in: Von Gott reden im Kontext der Armut. Dokumente der Ökumenischen Vereinigung von DritteWelt-Theologinnen und -Theologen 1976-1996, Freiburg i.Br. 1999, 33-46.

EKD, Zusammenleben gestalten. Ein Beitrag des Rates der EKD zu Fragen der Integration und des Zusammenlebens mit Menschen anderer Herkunft, Sprache oder Religion, EKD-T 76, 2002, http://www.ekd.de/EKD-Texte/ekd_texte_76_1.html. EKD, EKD-Denkschrift, Religiöse Orientierung gewinnen. Evangelischer Religionsunterricht als Beitrag zu einer pluralitätsfähigen Schule, November 2014 https://www.ekd.de/EKD-Texte/herausforderungen_religioes_weltanschaulichen_ vielfalt.html.

Espín, Orlando O., Die Theologie der Latinos/Latinas in den USA als Experiment in der Interkulturellen Theologie, in:Wolfgang GantKe/Thomas SchreijÄck (Hg.), Religionen im Kulturwandel zwischen Selbstannahme und Selbstaufgabe. Kontinental-kontextuelle Perspektiven, Forum Religionspädagogik Interkulturell 21, Berlin 2011, 33-48.

Estermann, Josef, Gott ist nicht europäisch, und die Theologie ist nicht abendländisch. Eine interkulturelle Dekonstruktion der abendländischen Theologie aufgrund der indigenen andinen Weisheit, in: IKTh 40 (2014), 186-200. 
EstermanN, Josef, Schritte zu einem polyzentrischen Religionsbegriff. Herausforderungen für die Religionspädagogik aus der Perspektive der interkulturellen philosophischen Diskussion, in:Thomas Schreı̈äck (Hg.), Christwerden im Kulturwandel. Analysen, Themen und Optionen für Religionspädagogik und Praktische Theologie. Ein Handbuch, Freiburg i.Br. 2001, 200-223.

Flake, SASKia/InA Schröder, Inklusive Pädagogik - Eine Herausforderung für die Religionspädagogik?!, in: KathaRina KAMMEYER ET AL. (Hg.), Inklusion und Kindertheologie, Inklusion - Religion - Bildung 1, Münster 2014, 30-64.

Freudenberger-Lötz, Petra, Theologische Gespräche mit Kindern, Erfahrungen - Beispiele - Anleitungen. Ein Werkstattbuch für die Sekundarstufe, München 2012. Gärtner, Claudia, Konfessioneller Religionsunterricht - kulturelle Vielfalt als Herausforderung eines auf Homogenität konzipierten und auf Identität zielenden Faches, in: Franz Gmainer-Pranzl et AL. (Hg.), Herausforderung Interkultureller Theologie, Beiträge zur Komparativen Theologie 26, Paderborn 2016, 89-104.

Gmainer-Pranzl, Franz, Der „Logos christlicher Hoffnung“ in globaler Verantwortung. Interkulturelle Theologie. Historische und hermeneutische Grundlagen, in: IKTh 40 (2014), 129-148.

Gruber, Judith, Theologie nach dem Cultural Turn. Interkulturalität als theologische Ressource, ReligionsKulturen 12, Stuttgart 2013.

JAHNEL, ClAudia, Interkulturelle Theologie und Kulturwissenschaft. Untersucht am Beispiel afrikanischer Theologie, Stuttgart 2016.

KAHL, Werner, Westafrikanische Bibelinterpretationen. Irritationen, Herausforderungen, Bereicherungen, in: BiKi 67 (2012), 149-153.

KIM, Uriah Y., Die Politik des Othering in Nordamerika und im Buch der Richter, in: Conc(D) 49 (2013), 152-161.

Kron, MARIA, Ausgangspunkt: Heterogenität. Weg und Ziel: Inklusion? Reflexionen zur Situation im Elementarbereich, Inklusion online-net 3/201oPositionspapier Religionspädagogik (http://akrk.eu/positionspapier-damit-der-religionsunterrichtin-deutschland-zukunftsfaehig-bleibt/) (10.1.2017).

KÜSTE R, VOLKER, Einführung in die Interkulturelle Theologie, Göttingen 2011.

Küster, Volker, Von fremden Christologien lernen. Das Antlitz Jesu Christi in Afrika, Asien und Lateinamerika neu entdecken, in: Helmut A. Müller (Hg.), Kultur, Religion und Glauben neu denken. Von der abrahamitischen Ökumene zur Ökumene der Weltreligionen, Berlin 2014, 65-84.

Link-Wieczorek, Ulrike, Ökumenische Herausforderungen für die Religionspädagogik, in: Theo-Web. Zeitschrift für Religionspädagogik 13 (2014) 2, 117-125.

Mecheril, PAUl, Einführung in die Migrationspädagogik, Weinheim/Basel 2004.

Mecheril, Paul /Thomas-Olalde, Oscar, Die Religion der Anderen. Anmerkungen zu Subjektivierungspraxen der Gegenwart, in: Brigit AllenbaCH ET AL. 
(Hg.), Jugend, Migration und Religion. Interdisziplinäre Perspektiven, Religion Wirtschaft - Politik 4, Zürich 2011, 35-66.

MÜLler-Fieberg, Rita, Interkulturelle Lernerfahrungen mit der Bibel, in: Franz Gmainer-Pranzl et AL. (Hg.), Herausforderung Interkultureller Theologie, Beiträge zur Komparativen Theologie 26, Paderborn 2016, 67-85.

Müller-Friese, Anita, Miteinander der Verschiedenen. Theologische Überlegungen zu einem integrativen Bildungsverständnis, FPDR 13, Weinheim 1996.

Offenberger, Bernhard, „To push the boundaries“. Die Grenzen des Wissens weiten. Ein Interview mit Musa W. Dube, in: BiKi 67 (2012), 160-163.

Positionspapier: Damit der Religionsunterricht in Deutschland zukunftsfähig bleibt. „Konfessionell, kooperativ, kontextuell - Weichenstellungen für einen zukunftsfähigen Religionsunterricht“ http://akrk.eu/positionspapier-damit-der-religionsunterricht-in-deutschland-zukunftsfaehig-bleibt/.

Reuter, Julia, Ordnungen des Anderen. Zum Problem des Eigenen in der Soziologie des Fremden, Bielefeld 2002.

Ricoeur, Paul, Geschichte und Wahrheit, München 1974.

Schweitzer, Friedrich/Thomas Schlag, Brauchen Jugendliche Theologie? Jugendtheologie als Herausforderung und didaktische Perspektive, NeukirchenVluyn 2011.

SEITZ, Klaus, Bildung im Horizont der Weltgesellschaft. Weltbürgerliche Erziehung im Zeitalter der Globalisierung, in: Thomas SchreıJäck (Hg.), Christwerden im Kulturwandel. Analysen, Themen und Optionen für Religionspädagogik und Praktische Theologie. Ein Handbuch, Freiburg im Breisgau 2001, 47-77.

Spivak, Gayatri Chakravorty, Can the Subaltern Speak? Postkolonialität und subalterne Artikulation (aus dem Englischen von AlexAnder Koskowicz und Stefan Nowotny), in: Boris Bude et Al. (Hg.), Es kommt darauf an, Texte zur Theorie der politischen Praxis 6, 18-118.

Streib, Heinz, Wie finden interreligiöse Lernprozesse bei Kindern und Jugendlichen statt? Skizze einer xenosophischen Religionsdidaktik, in: Peter Schreiner et AL. (Hg.), Handbuch interreligiöses Lernen, Gütersloh 2005, 230-243.

Vieregge, Dörthe, „Wenn der Typ korrekt ist, dann gucke ich nicht drauf, welche Religion er hat." Deutungen sozial benachteiligter Jugendlicher zum Umgang mit religiöser Differenz in der Lebenswelt, in: KATHARINA KAMMEYER ET AL. (Hg.), Inklusion und Kindertheologie, Inklusion - Religion - Bildung 1, Münster 2014, $82-93$.

Waldenfels, Bernhard, Grundmotive einer Phänomenologie des Fremden, Frankfurt am Main 2006.

Wolf, LARs, Ökumenischer Religionsunterricht - ein Beitrag zur Integration, in: PrTh 37 (2002), 16-22. 
Wrogemann, Henning, Religionen im Gespräch. Ein Arbeitsbuch zum interreligiösen Lernen, Stuttgart 2008.

Ziebertz, Hans-Georg/Stefan Heil/Andreas Prokopf (Hg.), Abduktive Korrelation. Religionspädagogische Konzeption, Methodologie und Professionalität im interdisziplinären Dialog, Münster 2003. 